\title{
ACF complex
}

National Cancer Institute

\section{Source}

National Cancer Institute. ACF complex. NCI Thesaurus. Code C20154.

The Drosophila gene Acf1 encodes AT P-dependent chromatin assembly factor large subunit, a component of the chromatin assembly complex involved in chromatin assembly/disassembly. Acf1 contains a PHD-finger and is able to modulate the internucleosomal spacing of chromatin by an ATP-dependent mechanism. Similar sequences have been identified in Caenorhabditis elegans, Homo sapiens, Mus musculus, and Saccharomyces cerevisiae. (from FlyBase 0027620 and NCl) 